\title{
Muscle and intensity based hamstring exercise classification in elite female track and field athletes: implications for exercise selection during rehabilitation
}

\author{
This article was published in the following Dove Press journal: \\ Open Access Journal of Sports Medicine \\ 26 June 2015 \\ Number of times this article has been viewed
}

\begin{abstract}
Panagiotis Tsaklis ${ }^{1,2}$
Nikos Malliaropoulos ${ }^{3-5,10}$

Jurdan Mendiguchia ${ }^{6}$

Vasileios Korakakis ${ }^{7-9}$

Kyriakos Tsapralis"

Debasish Pyne ${ }^{5}$

Peter Malliaras ${ }^{10}$

'Department of Physiotherapy, Laboratory of Biomechanics and Ergonomics, Alexander Technological Educational Institute of Thessaloniki, Thessaloniki, Greece; ${ }^{2}$ Department of Mechanical Engineering, Bioengineering, Massachusetts Institute of Technology, Cambridge, MA, USA; ${ }^{3}$ National Track and Field Centre, Sports Injury Clinic, Sports Medicine Clinic of SEGAS, ${ }^{4}$ Thessaloniki Sports Medicine Clinic, Thessaloniki, Greece; ${ }^{5}$ Rheumatology Department, Sports Medicine Clinic, Mile End Hospital, London, UK; ${ }^{6}$ Department of Physical Therapy, Zentrum Rehabilitation and Performance Center, Pamplona, Spain; ${ }^{7}$ Aspetar, Orthopaedic and Sports Medicine Hospital, Doha, Qatar; ${ }^{8}$ Faculty of Physical Education and Sport Science, University of Thessaly, Trikala, ${ }^{9}$ Hellenic Orthopaedic Manipulative Therapy Diploma, Athens, Greece; ${ }^{10} \mathrm{Centre}$ for Sports and Exercise Medicine, Queen Mary, University of London, London, UK; "K Tsapralis Isokinetic Medical Group, Bologna, Italy
\end{abstract}

Correspondence: Nikos Malliaropoulos Rheumatology Department, Sports Medicine Clinic, Mile End Hospital, Bancroft Road, London EI 4DG, UK Email contact@sportsmed.gr
Background: Hamstring injuries are common in many sports, including track and field. Strains occur in different parts of the hamstring muscle but very little is known about whether common hamstring loading exercises specifically load different hamstring components. The purpose of this study was to investigate muscle activation of different components of the hamstring muscle during common hamstring loading exercises.

Methods: Twenty elite female track and field athletes were recruited into this study, which had a single-sample, repeated-measures design. Each athlete performed ten hamstring loading exercises, and an electromyogram (EMG) was recorded from the biceps femoris and semitendinosus components of the hamstring. Hamstring EMG during maximal voluntary isometric contraction (MVIC) was used to normalize the mean data across ten repetitions of each exercise. An electrogoniometer synchronized to the EMG was used to determine whether peak EMG activity occurred during muscle-tendon unit lengthening, shortening, or no change in length. Mean EMG values were compared between the two recording sites for each exercise using the Student's $t$-test.

Results: The lunge, dead lift, and kettle swings were low intensity ( $<50 \% \mathrm{MVIC})$ and all showed higher EMG activity for semitendinosus than for biceps femoris. Bridge was low but approaching medium intensity, and the TRX, hamstring bridge, and hamstring curl were all medium intensity exercises ( $\geq 50 \%$ or $<80 \%$ MVIC). The Nordic, fitball, and slide leg exercises were all high intensity exercises. Only the fitball exercise showed higher EMG activity in the biceps femoris compared with the semitendinosus. Only lunge and kettle swings showed peak EMG in the muscle-tendon unit lengthening phase and both these exercises involved faster speed.

Conclusion: Some exercises selectively activated the lateral and medial distal hamstrings. Low, medium, and high intensity exercises were demonstrated. This information enables the clinician, strength and conditioning coach and physiotherapist to better understand intensity- and musclespecific activation during hamstring muscle rehabilitation. Therefore, these results may help in designing progressive strengthening and rehabilitation and prevention programs.

Keywords: hamstring injuries, exercise, rehabilitation

\section{Introduction}

Hamstring strain injuries are the most prevalent muscle injuries in track and field. ${ }^{1-4}$ Recent published evidence suggests that these injuries often cause prolonged symptoms and carry a high risk of reinjury, with devastating consequences for both performance and earning ability in elite athletes. ${ }^{1-3}$ 
During late swing phase of sprinting, the hamstring muscles act as decelerators of hip flexion and knee extension, ${ }^{5}$ and this is followed quickly by hip extension, constituting a stretch shorten cycle in the hamstring muscle. ${ }^{6}$ The most commonly reported mechanism of hamstring injury in track and field athletes involves high load eccentric contraction during the late swing phase (open kinetic chain).$^{5-8}$ In addition, kinetic and electromyogram (EMG) studies reveal that the hamstrings are most active and develop the greatest torque at the hip and knee during the late swing phase through to the mid stance phase of running..$^{5,6,8,9}$ Therefore, increasing hamstring strength may partly explain the success of recently proposed prevention programs. ${ }^{10-12}$ Despite published data showing that hamstring strains affect different segments of the hamstrings, $, 13,14$ little is known about hamstring activation during common prerehabilitation and rehabilitation hamstring exercises. ${ }^{15-18}$ The aim of this study was to evaluate individual hamstring muscle activation patterns during various hamstring exercises. Results of this study could be used to guide rehabilitation from injury to different hamstring muscles. ${ }^{19}$

\section{Materials and methods \\ Participant characteristics}

Twenty elite track and field athletes (from the Greek national team) volunteered for this study, and included ten sprinters, six long jumpers, and four triple jumpers. All were female, with a mean age of $22.8 \pm 3.7$ years, mean height of $169.6 \pm 5.5$ $\mathrm{cm}$, and a mean weight of $57.8 \pm 4.8 \mathrm{~kg}$. Individuals with a hamstring injury reported during the previous 6 months according to the clinic archives were excluded by the center lead sports medicine physician. The ethics committee of the Greek Track and Field Federation approved the study. All athletes provided written informed consent prior to participating in the study.

\section{Protocol}

Baseline testing involving EMG during maximal voluntary isometric contraction (MVIC) was performed, and participants were then instructed and familiarized with the ten exercises. After baseline testing, the participants performed the ten selected hamstring loading exercises, and a hamstring EMG was recorded during each exercise. These exercises were selected because they are commonly used in the prevention and management of hamstring injury. ${ }^{19}$ The limb measured was the dominant step leg for the jumpers and the preferred front block leg was considered dominant for the sprinters.

\section{EMG recording}

EMG recording was performed using the 8-channel Biomonitor ME6000 (Mega Electronics Ltd, Linton, UK). Prior to EMG recording, each participant's skin was shaved, sandpapered, and carefully cleaned with $70 \%$ alcohol. The biceps femoris longus and semitendinosus muscles were sampled. These muscles were chosen because they cover the most commonly affected components of hamstring injury., ${ }^{9,13}$ Disposable pregelled self-adhesive bipolar surface electrodes $(\mathrm{Ag} / \mathrm{AgCl}, 0.8 \mathrm{~cm}$ in diameter, Blue Sensor N-00-S, Medicotest A/S, Ølstykke, Denmark) were placed on the dominant leg with an interelectrode distance of $2 \mathrm{~cm}$ in accordance with SENIAM (Surface ElectroMyoGraphy for the Non-Invasive Assessment of Muscle) guidelines (http://www.seniam.org). This protocol was used to measure MVICs as well as EMG activity during the hamstring loading exercises.

\section{Normalization of MVIC and EMG}

The participants completed a warm-up that consisted of 10 minutes of submaximal cycling and 10 minutes of static stretching of the thigh muscles (quadriceps/hamstrings and adduction/abduction). A detailed presentation of the procedure for the MVICs and for familiarization with the isokinetic dynamometer (CSMI, Lumex, Ronkonkoma, NY, USA) was given, with participants lying prone and stabilized with knee flexion to $45^{\circ}$. The subjects performed two to three submaximal (50\% of maximum) isometric contractions to warm up and feel comfortable with the test that followed. During the test, they performed three MVICs with an isokinetic dynamometer. The EMG signal $(\mu \mathrm{V})$ from the two recorded channels was averaged. Standardized verbal encouragement and visual feedback from the monitor was given to each participant. Each isometric trial lasted 5 seconds. After a root-mean-square $(\mu \mathrm{V})$ adjustment, the trial with the highest averaged EMG signal was chosen and $80 \%$ of the value of the last 3 seconds was used as a reference for normalization of the EMG scores for each exercise. ${ }^{20}$

\section{Measurement of knee flexion}

Range of motion at the knee joint was measured during each exercise using a twin axis electrogoniometer (SG 150, data logger DL1001, Biometrics Ltd, Gwent, UK). The data were used to determine whether peak EMG during each exercise occurred during muscle-tendon unit (MTU) shortening, lengthening, or no change in length (ie, isometric). The electrogoniometer was connected and 
synchronized with the EMG device. Mechanical signals from the electrogoniometer were sampled at $200 \mathrm{~Hz}$ and converted into a digital signal.

\section{Hamstring loading exercise}

A senior sports physiotherapist supervised exercise for all participants and gave standardized instructions. Participants were familiarized with the proposed performance of the exercises, even though they were already familiar with some of these exercises and already used them during their regular practice. Participants performed one set of ten repetitions of each exercise in the same order (one to ten). Each exercise is detailed below. There was enough time between the testing exercises ( 5 minutes of rest) to relax and avoid possible accumulation of fatigue. In the event of a mistake during execution, the subject was required to relax for 3 minutes and then perform the same exercise again; however, no such incidents occurred during the study.

\section{Lunge}

From a standing start, each participants lunges forward so that their hip and knee are flexed to $90^{\circ}$, the back is upright, and there is no dynamic knee valgus (Figure 1). Participants

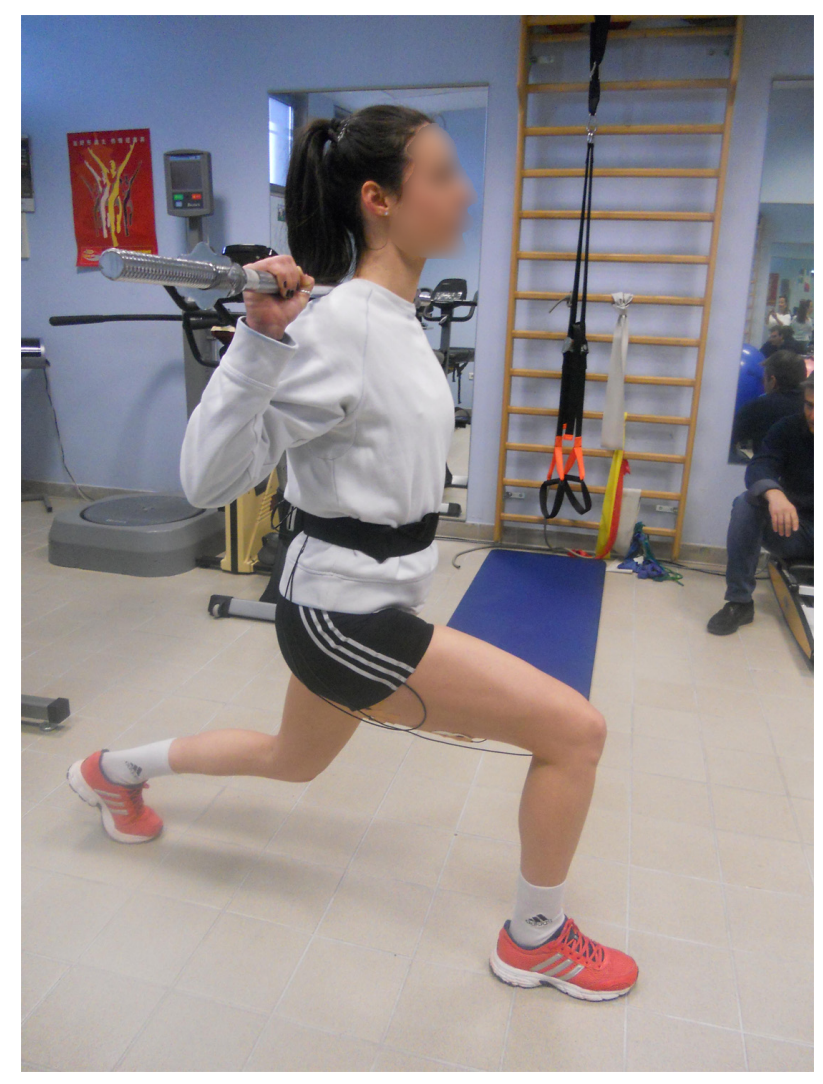

Figure I Lunge Exercise. are instructed to drop rapidly from the start to end position. This is a closed kinetic chain hip dominant exercise.

\section{Single leg Roman dead-lift T-drop}

Standing on one leg with the knee slightly bent, each participant maintains a neutral lumbar spine and slowly flexes to end range hip flexion (Figure 2). The back leg remains in neutral hip flexion-extension, and is moved backward as the trunk goes forward. This is a closed kinetic chain hip dominant exercise with limited knee flexion.

\section{Kettle bell swings}

A $12 \mathrm{~kg}$ kettle bell is used depending on ability and comfort (Figure 3). The participant bends forward to grip the kettle bell on the ground with both hands. The upper body at this point is parallel to the floor with the knees slightly flexed (approximately $10^{\circ}-15^{\circ}$ ). The participant is required to swing the kettle bell back between her legs forcefully then quickly reverse the direction with an explosive extension of the hips while swinging the kettle bell out to chest level; at this time, the hips and knees are extended with the participant standing upright. This is a closed kinematic chain hip dominant exercise.

\section{Bridge}

Participants start by lying in the supine position with arms by their side, knees bent to $90^{\circ}$, and feet flat on the ground. One leg is off the ground and placed over the opposite knee (Figure 4). The hips are lifted off the ground slowly until the knees, hips, and shoulders are in a straight line.

\section{TRX exercise}

The athlete is in the supine position and each foot is in the TRX straps (Figure 5). The pelvis is lifted and maintained

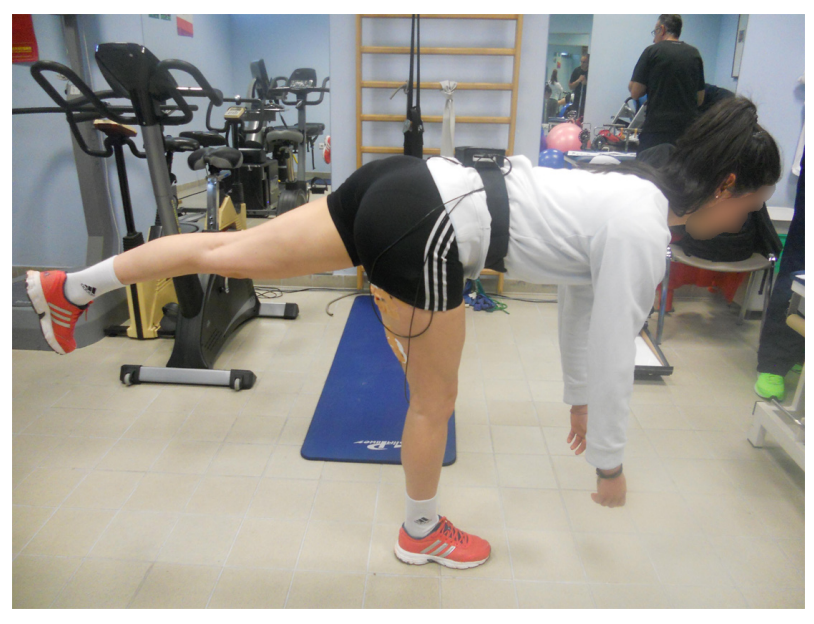

Figure 2 Single leg Roman dead-lift T-drop exercise. 


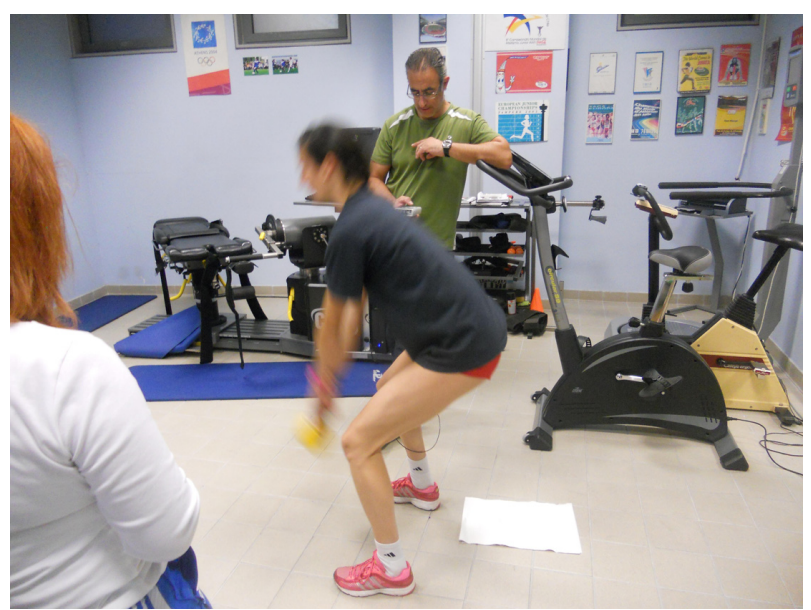

Figure 3 Kettle bell swing exercise.

in this position with the knees straight. Each knee is flexed alternately with a slow rhythm ensuring that the movement is controlled. One repetition equates to a single knee flexion on each side. This is an open kinetic chain knee dominant exercise.

\section{Hamstring bridge}

Participants start by lying in the supine position with arms by their side, knees bent, and heels on a chair (Figure 6). The hips are lifted off the ground slowly until the knees, hips, and shoulders are in a straight line. This position is held for 5 seconds.

\section{Curl}

Participants stand and perform slow concentric-eccentric knee flexion-hip extension against elastic band resistance (low load, $<2 \mathrm{~kg}$ ). Stability of the hip for the weight-bearing leg and neuromuscular control of the core is required in order to

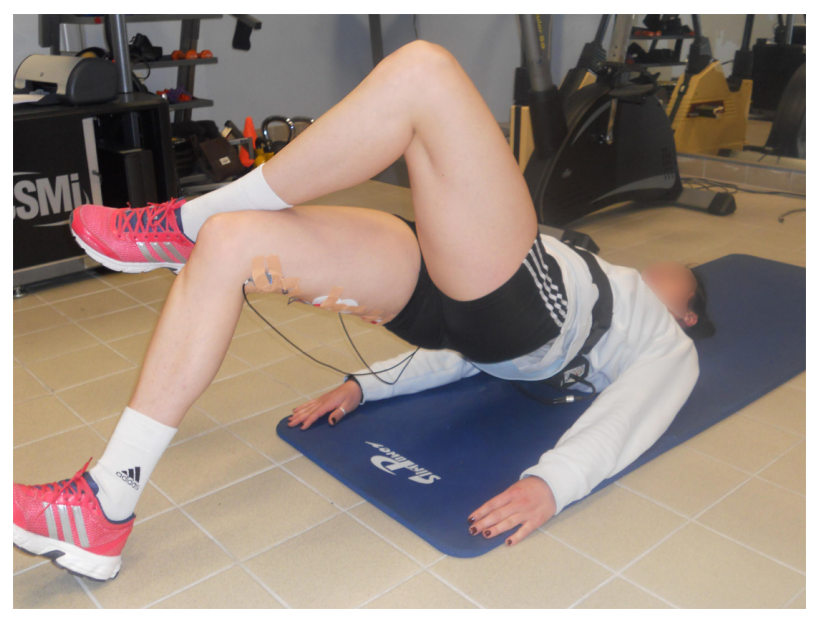

Figure 4 Bridge exercise.

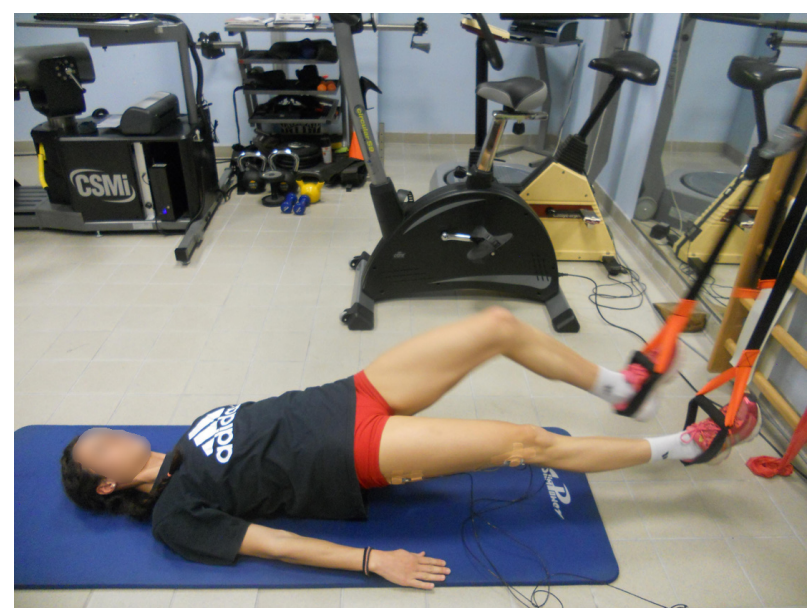

Figure 5 TRX exercise.

Note: TRX is a suspension trainer workout system that leverages gravity and your bodyweight.

counteract the torsional forces created at the trunk (Figure 7). This is an open kinetic chain knee dominant exercise.

\section{Nordic exercise}

Participants start by kneeling on a mat while a partner holds their ankles (Figure 8). The subject lowers their body forward in a slow and controlled manner whilst maintaining neutral hip flexion-extension. The subject lowers slowly as far as possible and then returns to the starting position. This is an open kinetic chain knee dominant exercise.

\section{Fitball flexion}

Participants start lying in a prone position (Figure 9). A fitball is held at the position of the gluteal muscles, and the subject flexes and hits the ball with the heel. The right and left legs are alternated as rapidly as possible. One repetition is hitting

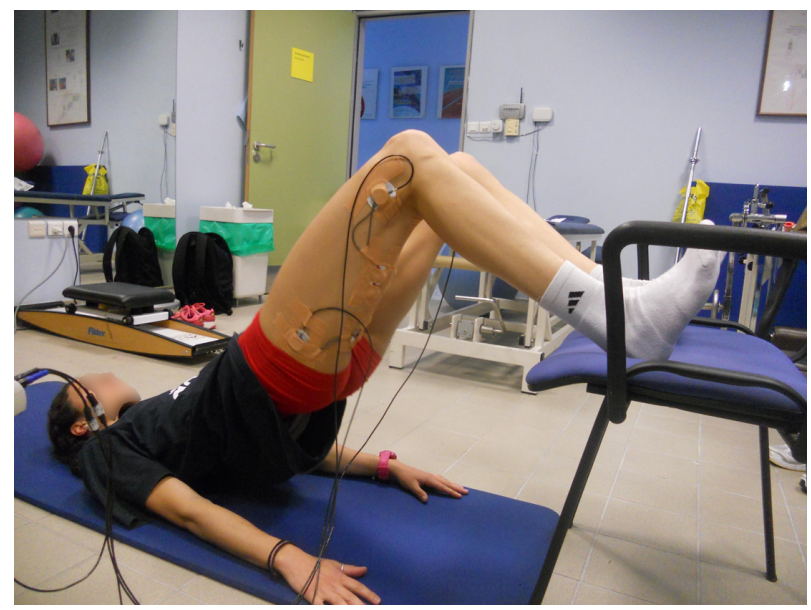

Figure 6 Hamstring bridge exercise. 


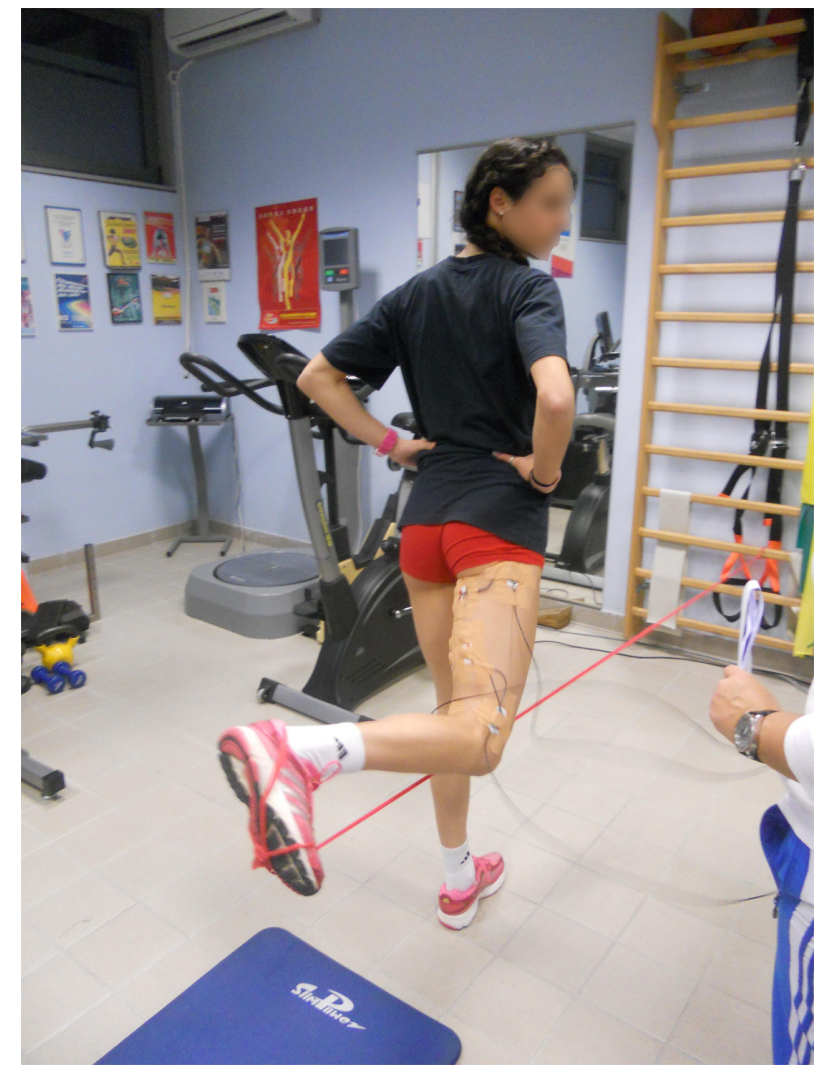

Figure 7 Curl exercise.

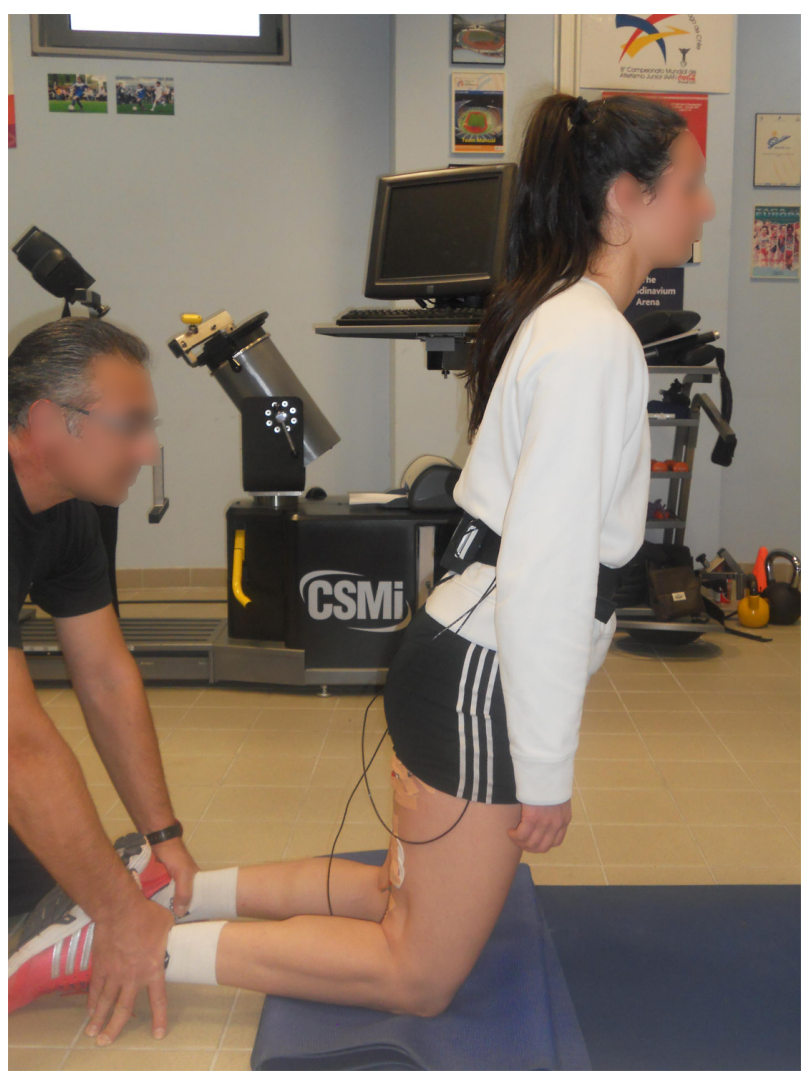

Figure 8 Nordic exercise.

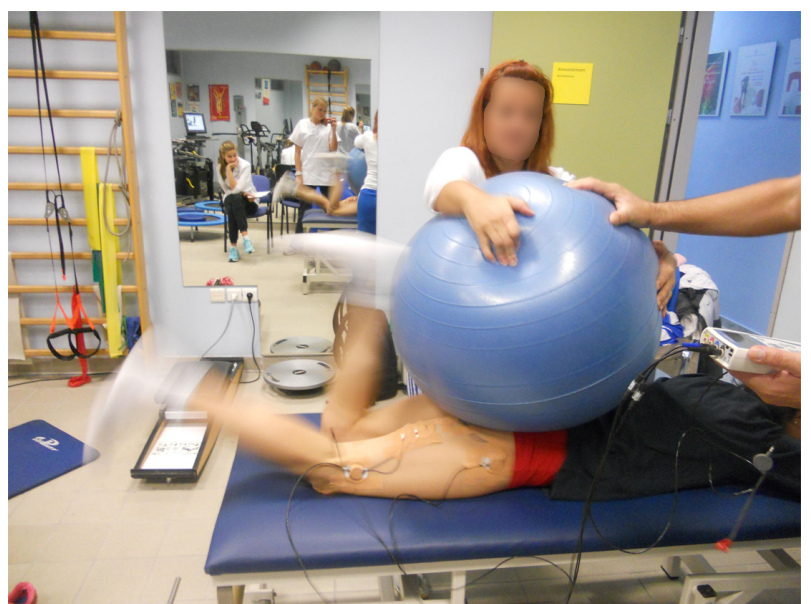

Figure 9 Fitball flexion exercise.

the ball once with each heel. This is an open kinetic chain knee flexion dominant exercise.

\section{Slide leg}

Participants start lying in the supine position on the floor with arms by their side, knees bent, and their heels on two pieces of rug which can easily slide over the floor (Figure 10). The heel on one side is used to weight-bear, with the pelvis off the ground, and the leg is straightened in a slow and controlled manner. The other leg is kept off the floor. When the knee of the working leg is straight, the leg is curled back. This is a closed kinetic chain knee flexion dominant exercise.

\section{Statistical analysis}

The mean EMG value was determined for each exercise by averaging the values for ten repetitions of each exercise. Mean EMG values were compared between the two recording

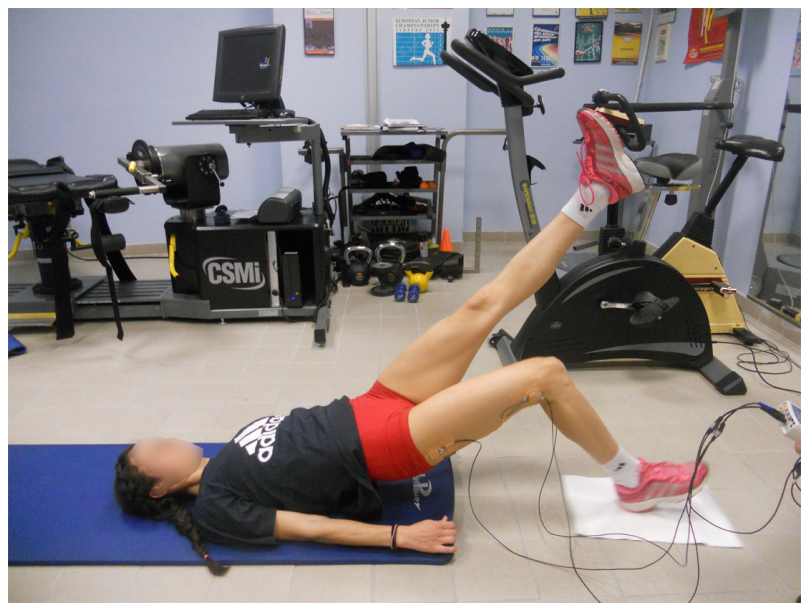

Figure 10 Slide leg exercise. 
sites for each exercise using the Student's $t$-test. The phase of movement (ie, MTU lengthening, shortening, or no change in length) in which peak EMG occurred was recorded. $P<0.05$ was considered to be statistically significant.

\section{Results}

Change in MTU length (lengthening, shortening, no change) for each exercise and whether it is closed or open kinetic chain is shown in Table 1. Peak EMG of the biceps femoris and semitendinosus muscles for each exercise is shown in Figure 11.

\section{Low intensity exercises ( $<50 \%$ MVIC)}

The lunge, single leg Roman dead-lift T-drop, and kettle swings were all closed kinetic chain and low intensity exercises, with greater activation of semitendinosus than biceps femoris. Peak EMG activity occurred during the MTU lengthening phase for the lunge and kettle swing and both lengthening and shortening phases for the dead lift. The onelegged bridge is also a closed kinetic chain exercise and was found to be low intensity but approaching medium intensity. Peak EMG in the single-legged bridge was during the MTU shortening phase and there was no bias toward activation of any specific hamstring component.

\section{Medium intensity exercises ( $\geq 50 \%$ or $<80 \%$ MVIC)}

The TRX, hamstring bridge, Nordic, and leg curl were all found to be medium intensity exercises. However, the Nordic exercise was found to be high intensity when greater lower range of motion was achieved. The intensity depends on the ability of the participant to increase lowering range. The Nordic is an open kinetic chain exercise with peak EMG during the MTU lengthening and shortening phases, without preferential hamstring activation. The TRX and leg curl are both open kinetic chain exercises. The TRX showed peak EMG activity when the MTU was isometric, whereas peak EMG in the leg curl was during the MTU shortening phase. Neither exercise demonstrated preferential muscle activation. The hamstring bridge is a closed chain exercise which showed peak EMG in the MTU shortening phase but no bias toward specific hamstring activation.

\section{High intensity exercises ( $\geq 80 \%$ of MVIC)}

The fitball flexion and slide leg exercises were found to be high intensity. Fitball flexion exercise is a track and field sports-specific open kinetic chain exercise and showed peak EMG activity during the MTU shortening phase. It was the only exercise causing greater activation of biceps femoris than semitendinosus. The slide leg is an open kinetic chain exercise with the highest intensity of all exercises. Peak EMG was shown during the MTU lengthening and shortening phases with no preferential hamstring activity.

The main finding of our study was that commonly prescribed hamstring exercises can be divided into semitendinosus-dominant and biceps femoris longusdominant, and can be classified into high, medium, or low according to their activation intensity.

During execution of the lunge, kettle swing, and single leg Roman dead-lift T-drop, the semitendinosus muscle is preferentially targeted. However, the intensity level of these exercises can be categorized as low. Previous authors have also found greater activation of semitendinosus vs biceps femoris for the single leg Roman dead-lift T-drop with 12 repetition maximum load and kettle bell swings. ${ }^{18,21}$ These findings may be partially explained by the fact that the semitendinosus is a fusiform muscle with parallel fibers and long fiber lengths, whereas biceps femoris and semimembranosus have a unipennate and bipennate arrangement. ${ }^{22,23}$ Given these characteristics, semitendinosus may be more sensitive

Table I Contraction type and kinetic chain

\begin{tabular}{|c|c|c|c|c|c|}
\hline & No change & Shortening & Lengthening & Open kinetic chain & Closed kinetic chain \\
\hline \multicolumn{6}{|l|}{ Lunge } \\
\hline \multicolumn{6}{|c|}{ Single leg Roman dead-lift T-drop } \\
\hline \multicolumn{6}{|c|}{ Kettle swing } \\
\hline \multicolumn{6}{|l|}{ Bridge } \\
\hline \multicolumn{6}{|l|}{ Bridge } \\
\hline \multicolumn{6}{|l|}{$\operatorname{TRX}$} \\
\hline \multicolumn{6}{|l|}{ Curl } \\
\hline \multicolumn{6}{|l|}{ Nordic } \\
\hline \multicolumn{6}{|l|}{ Fitball flexion } \\
\hline Slide leg & & & & & \\
\hline
\end{tabular}

Notes: TRX is a Suspension Trainer workout system that leverages gravity and your bodyweight. Dark grey areas refer to the type of muscle contraction which recorded the higher EMG activity in every exercise and the light grey refer to the Kinetic Chain through which, each exercise is performed.

Abbreviation: EMG, electromyogram. 


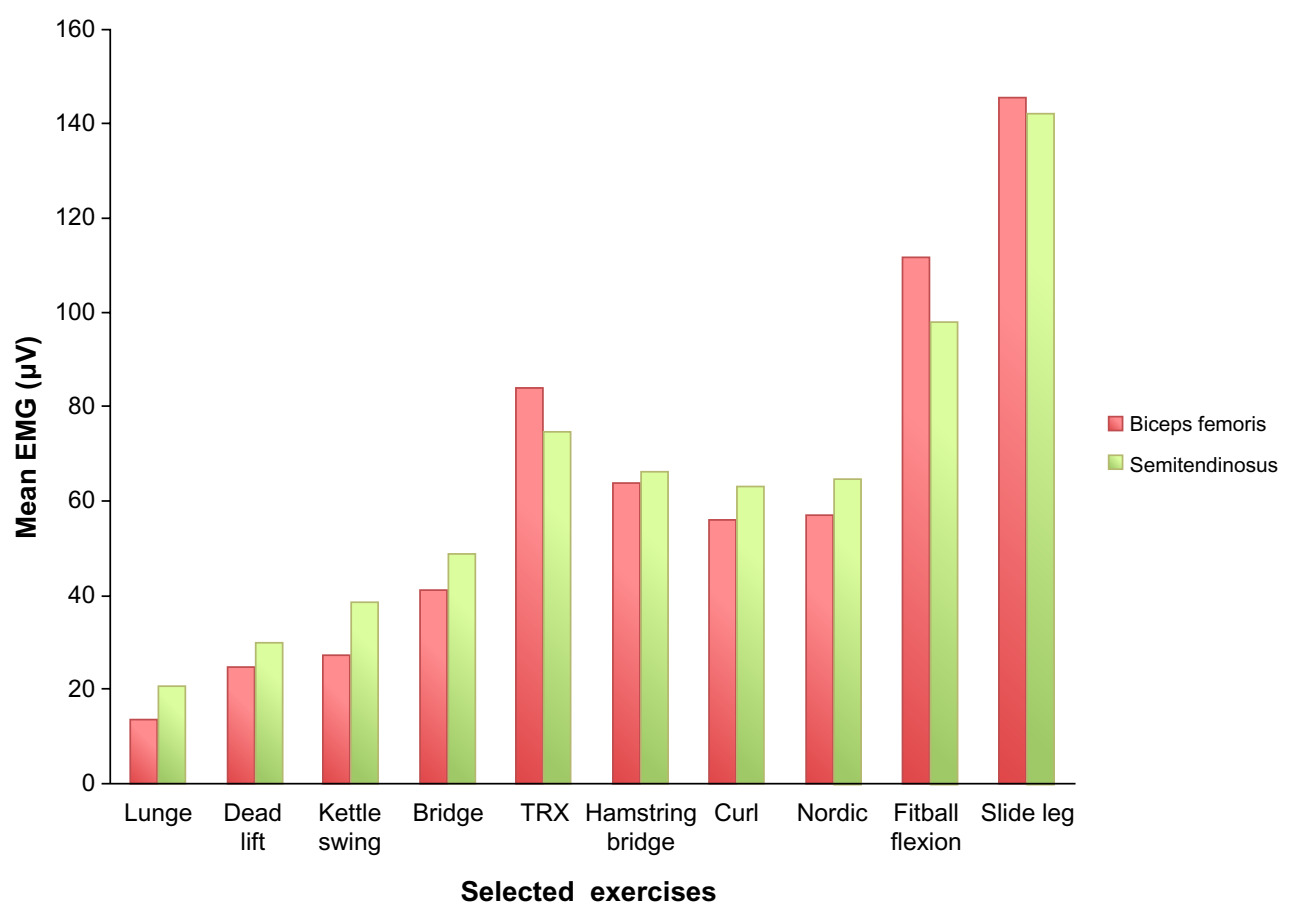

Figure I I Hamstring muscle component EMG (percent maximal voluntary isometric contraction) for each exercise.

Note: TRX is a Suspension Trainer workout system.

Abbreviation: EMG, electromyogram.

to exercise involving a large change in MTU length, such as when hip flexion actions are required. ${ }^{18}$

The unique exercise in this experiment showing selective activation of biceps femoris was fitball flexion, a common track and field sports-specific open kinetic chain exercise, which showed peak EMG activity during the MTU shortening phase. Previous research comparing bíceps femoris activity between exercises is conflicting. Some authors have found that the prone lying leg curl, ${ }^{18}$ hip hyperextension, ${ }^{18}$ and single leg Roman dead-lift T-drop ${ }^{24}$ lead to a greater biceps response. Conversely, McAllister et $\mathrm{al}^{25}$ found that the activity of semitendinosus was higher in all exercises studied, including both knee dominant (prone leg curl and glute-ham raise) and hip dominant exercises (good morning and Roman dead lift).

There were several exercises that showed similar EMG activation for both the medial and lateral components of the hamstring complex. These included exercises with low (single leg bridge), medium (curl, hamstring bridge) and high (Nordic, slide leg) activation levels. Iga et $\mathrm{al}^{17}$ also found no difference in medial and lateral hamstring EMGs during the Nordic exercise. In contrast, Mendiguchia et al,26 found that Nordic hamstring exercise created greater damage response (indicating training stimulus, measured with T2 MRI) in biceps femoris short head proximal section compared with the medial hamstring group. Lack of complete agreement between EMG (electrical activation) and T2 (metabolic activity) shift recording can be indicative of the different physiological basis of each measure and may explain the differential activation and damage patterns of the lateral vs medial hamstrings during performance of Nordic hamstring exercises. ${ }^{27}$ This series of exercises with similar EMG activity in different hamstring components may be used to progressively increase activation and loading of the hamstring muscle group during rehabilitation. Low intensity exercises may be particularly useful in the early period following acute muscle injury.

Hamstring strains may occur proximally or distally, 2,9,13 and generally involve eccentric muscle action, such as knee extension (eg, leg deceleration in terminal swing) or hip flexion (eg, picking up the ball in some football codes).,2,28,29 Nordic and slide leg exercises involve peak EMG in MTU lengthening combined with knee extension, whereas lunge, kettle swings, and the dead lift involve peak EMG in MTU lengthening and hip flexion. High eccentric load may be important to replicate sports loads and shift the optimal length to longer ranges to guard against injury. ${ }^{30}$ The two exercises with peak EMG during only the MTU lengthening phase involved speed and are classified as low intensity exercises (kettle swings, lunge). Speed or greater external load may be prerequisites for peak EMG during the MTU lengthening phase, given the generally lower EMG activity with eccentric 
compared with concentric muscle action. ${ }^{21,25}$ In a rehabilitation context, speed needs to be considered in relation to the symptoms and healing phase.

An important finding was that high activation of the all hamstring muscle components can be achieved without the need for heavy external loading (ie, gym weights and machines) and this is best demonstrated by the Nordic and slide leg exercises. Load is often applied in a rehabilitation context, and this may change the muscle activation profile of each exercise. For example, hamstring curls are often progressed to heavy load in a hamstring curl machine and this is certainly likely to increase activation of the hamstring. For example, Ebben et al ${ }^{16}$ found a higher peak normalized EMG than our study for the curl (81\% vs 60\%) and dead lift (49\% vs 22\%), probably because the load was higher (six repetition maximum compared with no loading in our study).

The two exercises with the highest EMG activation were open chain, whereas the three exercises with the lowest EMG activation were closed chain, confirming a previous report by Ebben et al. ${ }^{16}$ This highlights the fact that although closed change exercises may replicate function and be important in developing neuromuscular specificity for load-bearing functional tasks, there should be a place for open chain exercises to maximize hamstring loading, especially in the absence of a heavy external load, as in this study. It also challenges the view of progression to functional closed kinetic chain exercise and reinforces the fact that low activation closed kinetic chain exercises (eg, lunge) can be safely applied in the early phases of rehabilitation.

This study has several limitations. We only assessed women, so the findings cannot be generalized to male track and field athletes. All exercises were performed in the same order (ie, one to ten), so fatigue may have influenced the EMG data during the latter loading exercises. To minimize this risk, participants only performed one set of each exercise and exercises were performed with little or no load. As exercises were performed with low load, they may not replicate clinical loading practices, especially in the latter phases and in prevention programs, and this should be addressed in future studies. We identified MTU lengthening and shortening phases but were not able to identify eccentric and concentric muscle action, so we cannot be sure of the hamstring muscle action at peak EMG. Despite this, it would seem most important to replicate the loading range of motion and MTU length change, so we believe our data are clinically applicable.

Low, medium, and high intensity exercises were demonstrated. This information provides the clinician, strength and conditioning coach, and/or physiotherapist with a better understanding of intensity- and site-specific activation of the hamstrings during common posterior thigh loading exercises. Therefore, these results may help in the design of progressive strengthening, rehabilitation, and prevention programs.

\section{Author contributions}

All authors contributed to conception and design of the study, interpretation of the data, as well as drafting and writing the manuscript and final approval. PM and NM performed the statistical analysis. NM, JM, PT were involved in acquisition of the data. All authors agree to be accountable for all aspects of the work.

\section{Disclosure}

This paper received no specific grant from any funding agency in the public, commercial or not-for-profit sectors. The authors report no conflicts of interest in this work.

\section{References}

1. Alonso JM, Tscholl PM, Engebretsen L, Mountjoy M, Dvorak J, Junge A. Occurrence of injuries and illnesses during the 2009 IAAF World Athletics Championships. Br J Sports Med. 2010;44(15):1100-1105.

2. Askling C, Saartok T, Thorstensson A. Type of acute hamstring strain affects flexibility, strength, and time to return to pre-injury level. $\mathrm{Br} J$ Sports Med. 2006;40(1):40-44.

3. Malliaropoulos N, Isinkaye T, Tsitas K, Maffulli N. Reinjury after acute posterior thigh muscle injuries in elite track and field athletes. $\mathrm{Am} \mathrm{J}$ Sports Med. 2011;39(2):304-310.

4. Malliaropoulos N, Papacostas E, Kiritsi O, Papalada A, Gougoulias N, Maffulli N. Posterior thigh muscle injuries in elite track and field athletes. Am J Sports Med. 2010;38(9):1813-1819.

5. Thelen DG, Chumanov ES, Hoerth DM, et al. Hamstring muscle kinematics during treadmill sprinting. Med Sci Sports Exerc. 2005;37(1): $108-114$.

6. Yu B, Queen RM, Abbey AN, Liu Y, Moorman CT, Garrett WE. Hamstring muscle kinematics and activation during overground sprinting. J Biomech. 2008;41(15):3121-3126.

7. Heiderscheit BC, Hoerth DM, Chumanov ES, Swanson SC, Thelen BJ, Thelen DG. Identifying the time of occurrence of a hamstring strain injury during treadmill running: a case study. Clin Biomech. 2005; 20(10):1072-1078.

8. Schache AG, Wrigley TV, Baker R, Pandy MG. Biomechanical response to hamstring muscle strain injury. Gait Posture. 2009;29(2): 332-338.

9. Connell DA, Schneider-Kolsky ME, Hoving JL, et al. Longitudinal study comparing sonographic and MRI assessments of acute and healing hamstring injuries. AJR Am J Roentgenol. 2004;183(4):975-984.

10. Arnason A, Andersen TE, Holme I, Engebretsen L, Bahr R. Prevention of hamstring strains in elite soccer: an intervention study. Scand J Med Sci Sports. 2008;18(1):40-48.

11. Askling C, Karlsson J, Thorstensson A. Hamstring injury occurrence in elite soccer players after preseason strength training with eccentric overload. Scand J Med Sci Sports. 2003;13(4):244-250.

12. Petersen J, Thorborg K, Nielsen MB, Budtz-Jørgensen E, Hölmich P. Preventive effect of eccentric training on acute hamstring injuries in men's soccer: a cluster-randomized controlled trial. Am J Sports Med. 2011;39(11):2296-2303. 
13. Koulouris G, Connell DA, Brukner P, Schneider-Kolsky M. Magnetic resonance imaging parameters for assessing risk of recurrent hamstring injuries in elite athletes. Am J Sports Med. 2007;35(9):1500-1506.

14. Woods C, Hawkins RD, Maltby S, Hulse M, Thomas A, Hodson A. The Football Association Medical Research Programme: an audit of injuries in professional football - analysis of hamstring injuries. $\mathrm{Br} J$ Sports Med. 2004;38(1):36-41.

15. Ditroilo M, De Vito G, Delahunt E. Kinematic and electromyographic analysis of the Nordic hamstring exercise. J Electromyogr Kinesiol. 2013;23(5):1111-1118.

16. Ebben WP. Hamstring activation during lower body resistance training exercises. Int J Sports Physiol Perform. 2009;4(1):84-96.

17. Iga J, Fruer CS, Deighan M, Croix MD, James DV. Nordic hamstrings exercise - engagement characteristics and training responses. Int $J$ Sports Med. 2012;33(12):1000-1004.

18. Zebis MK, Skotte J, Andersen CH, et al. Kettlebell swing targets semitendinosus and supine leg curl targets biceps femoris: an EMG study with rehabilitation implications. Br J Sports Med. 2013;47(18):1192-1198.

19. Malliaropoulos N, Mendiguchia J, Pehlivanidis H, et al. Hamstring exercises for track and field athletes: injury and exercise biomechanics, and possible implications for exercise selection and primary prevention. Br J Sports Med. 2012;46(12):846-851.

20. De Luca CJ. The use of surface electromyography in biomechanics. J Appl Biomech. 1997;13(2):135.

21. Wright GA, Delong TH, Gehlsen G. Electromyographic activity of the hamstrings during performance of the leg curl, stiff-leg deadlift, and back squat movements. J Strength Cond Res. 1999;13(2):168-174.
22. Kumazaki T, Ehara Y, Sakai T. Anatomy and physiology of hamstring injury. Int J Sports Med. 2012;33(12):950-954.

23. Woodley SJ, Mercer SR. Hamstring muscles: architecture and innervation. Cells Tissues Organs. 2005;179(3):125-141.

24. Ono T, Higashihara A, Fukubayashi T. Hamstring functions during hip-extension exercise assessed with electromyography and magnetic resonance imaging. Res Sports Med. 2011;19(1):42-52.

25. McAllister MJ, Hammond KG, Schilling BK, Ferreria LC, Reed JP Weiss LW. Muscle activation during various hamstring exercises. J Strength Cond Res. 2014;28(6):1573-1580.

26. Mendiguchia J, Arcos AL, Garrues MA, Myer GD, Yanci J, Idoate F. The use of MRI to evaluate posterior thigh muscle activity and damage during Nordic hamstring exercise. J Strength Cond Res. 2013;27(12): 3426-3435.

27. Kubota J, Ono T, Araki M, et al. Relationship between the MRI and EMG measurements. Int J Sports Med. 2009;30(07):533-537.

28. Brughelli M, Cronin J. Preventing hamstring injuries in sport. Strength Cond J. 2008;30(1):55-64.

29. Chumanov ES, Schache AG, Heiderscheit BC, Thelen DG. Hamstrings are most susceptible to injury during the late swing phase of sprinting. Br J Sports Med. 2012;46(2):90.

30. Brockett CL, Morgan DL, Proske U. Human hamstring muscles adapt to eccentric exercise by changing optimum length. Med Sci Sports Exerc. 2001;33(5):783-790.
Open Access Journal of Sports Medicine

\section{Publish your work in this journal}

Open Access Journal of Sports Medicine is an international, peer-reviewed, open access journal publishing original research, reports, reviews and commentaries on all areas of sports medicine. The manuscript management system is completely online and includes a very quick and fair peer-review system.

\section{Dovepress}

Visit http://www.dovepress.com/testimonials.php to read real quotes from published authors. 